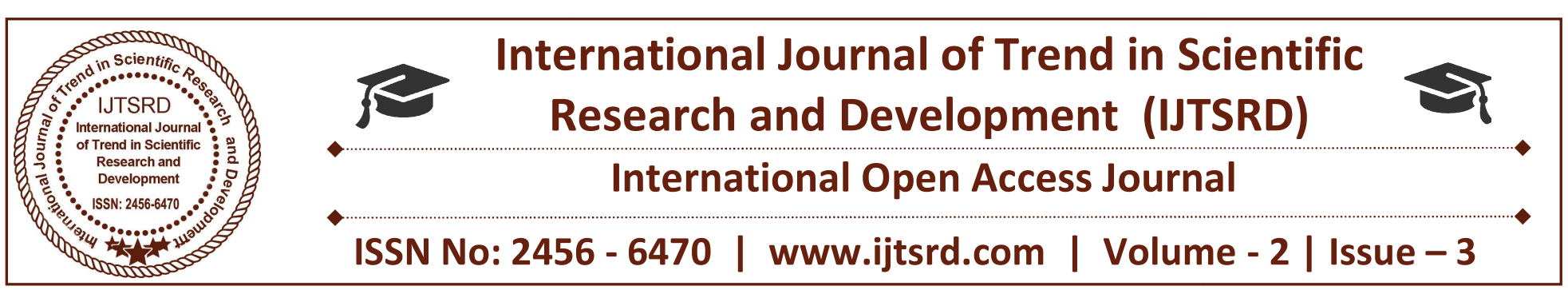

\title{
Evaluation of Bioactivecomponents and Antistaphylococcal Activitiesof Ethyl Acetate and Dicloromethanefractions of Moringa Oleifera Root Bark on Clinical Isolates of Methicillin Resistant
} Staphylococcus Aureus

Agboke A. A.

Department of Pharmaceutics and Pharmaceutical Technology, University of Uyo, Uyo, Akwa Ibom State, Nigeria
Attama A. A.

Department of Pharmaceutics and Pharmaceutical Microbiology, University of Nigeria, Nsukka, Enugu state, Nigeria

\section{ABSTRACT}

This study evaluated the phytochemical components, Bioactive compounds (GC-MS) and antistaphylocochal activities of ethyl acetate and dichloromethane fractions of Moringa oleifera root bark. MRSA (Methicillin resistance staphylococcus aureus) was used for the antistaphylococcal activities. The isolates from 3 different hospitals in south-east geopolitical region of Nigeria were confirmed by coagulase/staphylase test using Oxoid ${ }^{\circledR}$ reagents kits (DR0595A). MRSA confirmation was done using Oxoid $^{\circledR}$ DR0900 penicillin binding protein (pbp2) latex agglutination test kits. Pulverised Moringa oleifera root bark was extracted with methanol using Soxhlet extractor to obtain methanol crude extract (ME). ME was adsorbed on Silical gel (60-200 mesh) and eluted with ethyl acetate solvent to get ethyl acetate fraction (EAF) and also with dichloromethane solvent to get dichloromethane fraction (DMF). Qualitative phytochemical analyses of the EAF and DMF were carried out using standard procedures. The antistaphylococcal activities of EAF and DMF were evaluated on the MRSA, the minimum inhibitory concentrations (MICs) and minimum bactericidal concentrations (MBCs) were recorded and compared with the standard disc antimicrobial test results. The two extract fractions were analyzed using gas chromatographic-mass spectrometry (GC-MS) for their bioactive compounds. Statistical analysis was done with ANOVA followed by Duncan post Hoc test using SPSS v 17 software. The results obtained showed: DMF MIC $(8.0 \pm 1.1$ to $10 \pm 0.5 \mathrm{mg} / \mathrm{ml})$ and $\operatorname{MBC}(8.0 \pm 0.5$ to $10 \pm 0.5 \mathrm{mg} / \mathrm{ml}) ; \mathrm{EAF}: \mathrm{MIC}(5.0 \pm$ 1.1 to $8.0 \pm 0.5 \mathrm{mg} / \mathrm{ml})$ and $\mathrm{MBC}(5.0 \pm 0.5$ to $8.0 \pm$ $0.5 \mathrm{mg} / \mathrm{ml}$. Phytochemical analysis of the extract fractions showed: DMCwith concentrations high in Resins followed by steroids, fats and oil with traces of alkaloids, terpenoids and flavonoids, while EAF has high concentrations of Flavonoids, with traces of glycosides, terpenoids, steroids and carbohydrates. The GC-MS analysis revealed the bioactive components from the two solvents. Ethyl acetate (EAF) fraction is more potent than dichloromethane fraction; this indicates that the most active compound against the MRSA can be isolated from the EAF.

Keywords: Activities, Antimicrobial, Bioactive, Components, Compounds, Dichloromethane, Ethyl acetate, MRSA, Phytochemical

\section{INTRODUCTION}

The evaluation of various plant products according to their traditional uses and medicinal value based on their therapeutic efficacy leads to the discovery of newer and recent drugs for treat ingvarious ailments [1]. This fact forms the basis for the development of new drugs from various plant sources. One of such plants of medicinal value is Moringa olifera, belonging to the family Moringaceae, commonly known as 'sahajan' in Hindi, Horse radish in English. It is a small, fast, growing, evergreen, or deciduous 
tree that usually grows up to 10 or $12 \mathrm{~m}$ in height. It is distributed among Sub Himalayan Tracts, Assam, Bengal and Peninsular India [1]. Various properties are attributed to it like antispasmodic, diuretic, expectorant and abortifacient [2].

\section{Moringa oleifera}

The Horticultural College \& Research Institute of Tamil Nadu Agricultural University has released two improved annual moringa varieties (PKM1, PKM2) within a span of 10 years, for commercial cultivation $[3,4]$. The folklore claims and ancient literature report moringa to be an abortifacient antidote, antirheumatic, bactericide, diuretic, ecbolic, emetic, expectorant, purgative, rubefacient, stimulant, tonic, vermifuge and vesicant [5-7]. (Pharma Products Pvt Ltd, Thayavur, India) and Livospin (Herbals APS Pvt. Ltd., Patna, India), which are available for a variety of ailments [8]. Ayurvedic preparations include Ratnagiri Rasa, Sarasvata Ghrta, Sudarsana churna, Sarsapadi Pralepa, Visatimduka Taila etc $[4,5]$.

Until now, only a very few attempts have been made to compile the myriad of potential uses of this "miracle tree". In view of a number of recent findings of ethnopharmacological importance, an updated appraisal was much needed. The present research is an attempt to explore the claims so far and prepare the ground for development of effective novel herbal formulations of $M$. oleifera. in the treatment of infections caused by much dreaded Methicilin resitant Staphylococcus aureus

\section{Antibacterial and antifungal activities}

Moringaroots have antibacterial activity [9] and are reported to be rich in antimicrobial agents. These are reported to contain an active antibiotic principle, pterygospermin, which has powerful antibacterial and fungicidal effects. A similar compound is found to be responsible for the antibacterial and fungicidal effects of its flowers [10]. The root extract also possesses antimicrobial activity attributed to the presence of 4$\alpha$-L-rhamnosyloxybenzyl isothiocyanate [11]. The aglycone of deoxy-niazimicine [N-benzyl, S-ethyl thioformate] isolated from the chloroform fraction of an ethanol extract of the root bark was found to be responsible for the antibacterial and antifungal activities [12]. The bark extract has been shown to possess antifungal activity [13], while the juice from the stem bark showed antibacterial effect against Staphylococcus aureus [14]. The fresh leaf juice was found to inhibit the growth of microorganisms
[Pseudomonas aeruginosa and Staphylococcus aureus], pathogenic to man [15].

The seeds also possess antimicrobial properties [16, 17] reported that a recombinant protein in the seed is able to flocculate Gram-positive and Gram-negative bacterial cells. In this case, microorganisms can be removed by settling in the same manner as the removal of colloids in properly coagulated and flocculated water [17]. On the other hand, the seeds may also act directly upon microorganisms and result in growth inhibition. Antimicrobial peptides are thought to act by disrupting the cell membrane or by inhibiting essential enzymes.

It was reported that the seeds could inhibit the replication of bacteriophages $[17,18,19]$. The antimicrobial effects of the seeds are attributed to the compound $4[\alpha-\mathrm{L}$-rhamnosyloxy] benzyl isothiocynate [19].

\section{MATERIALS AND METHODS}

\section{MATERIALS}

Clinical isolates: The clinical isolates used include Staphylococcus aureus, methicilin resistant Staphyloccocus aureus, from Bishop Shanahan Hospital, Nsukka, University of Nigeria Teaching Hospital, Ituku/Ozalla Enugu state and Federal Medical Centre Abakaliki, Ebonyi State.

Media : Nutrient Agar (Fluka) Sigma Aldrich UK, Mueller-Hinton Agar (MHA), Oxoid Ltd, England, Mannitol Salt Agar (MSA), Oxoid Ltd, England.

Reagents: PBP2a or PBP2' test kit dr0900a lot. no. 130422. Oxoid Ltd, Japan, staphylase test kit dr0595a., (Oxoid Ltd, Wade Road, Basingstoke, Hants, RG24, UK), Oxoid antimicrobial susceptibility test discs. Hydrogen peroxide $\left(\mathrm{H}_{2} \mathrm{O}_{2}\right)$, Dimethylsulfoxide (DMSO), distilled water, silical gel, (Titan Biotech Ltd, India). 0.5 McFarland turbidity standard.

Solvents: Methanol, (Sigma Aldrich, U.K), ethyl acetate, (Sigma Aldrich U.K), dichloromethane (Sigma Aldrich U.K).

Equipment :Test tubes, Petri-dish, pipette, micropipette, micro centrifuge tube, measuring cylinder, flat bottom flask, Soxhlet extractor, glass chromatographic column, autoclave, refrigerator, cotton wool, weighing balance, foil, sterile loops and swabs, incubator, antibiotic disc dispensers, GC-MS 
equipment with Agilent technologies 7890B for GC systems and Agilent technologies 5975 series for MS system.[34].

Collection, authentication and processing of plant materials: The root of Moringa oleifera was collected from Nsukka Local Government Area, Enugu State, Nigeria. The plant materials were identified and authenticated by a Botanist at the Biological Science Department, University of Nigeria, Nsuka. Confirmation of taxonomic identity of the plants was achieved by Mrs. Immanuela Udoma by comparison with voucher specimens kept at the Herbarium of the Department of Pharmacognosy, Faculty of Pharmacy, University of Uyo, and use of documented literature [21]. The plant materials were air-dried in the laboratory for four weeks. The dried samples were grinded to coarse powder with a mechanical grinder; the powdered was stored for future use.

Extraction of the root extract: The pulverized root of Moringa oleifera (3 kg) was extracted with 20 litres of methanol for 4 hours using Soxhlet extraction technique to yield methanol crude extract (ME) using established standard procedures by Harborne, Iwu, Trease and Evans [21-23].

The methanol crude extract (ME) was concentrated in-vacuo using rotary evaporator and yielded percentage was calculated. The dried extract (ME) (850.60 g) was adsorbed on silica gel (60-200 mesh) in a glass column, was then eluted in succession with dichloromethane and ethyl acetate to yield dichloromethane fraction (DF), ethyl acetate fraction (EAF). [21-23].

\section{Qualitative phytochemical analysis}

The ethyl acetate and dichloromethane fractions of the Moringa oleifera root bark was subjected to phytochemical tests using established standard procedures by Harborne, Iwu, Trease and Evans [2123].

\section{Penicilin-binding protein $\left(\right.$ PBP2 $\left.^{1}{ }^{\top}\right)$ latex agglutination test for MRSA confirmation}

PBP2' extraction procedure as recommended by the Manufacturer (Oxoid,).
Test was performed only on Staphylococcus species (Gram + positive cocci).

Four drops of Extraction Reagent 1 was added to a micro centrifuge tube, an approximately $1.5 \times 10^{9}$ (3-5 $\mu l)$ cells was then suspended into the micro centrifuge tube to obtain a very turbid suspension. The tube was placed into a water bath at temperature over $95^{\circ} \mathrm{C}$ and allowed to heat for three minutes, it was removed and allowed to cool to room temperature before adding a drop of extraction reagent 2 and the mixture was vigorously shook to obtain homogenous mixture. The mixture was centrifuged at $3000 \mathrm{rpm}$ at $15 \mathrm{~cm}$ rotation radius for 5 minutes to obtain a supernatant solution containing the extracted PBP2a for MRSA.

Latex agglutination procedure For each supernatant to be tested, one circle of the test card was labeled ' $\mathbf{T}$ ' for testing with Test Latex and another with ' $\mathbf{C}$ ' for Control Latex. The latex reagent was properly mixed by inversion several times and a drop of test Latex or Control Latex was added to each labeled circle accordingly. $50 \mu \mathrm{l}$ of supernatant was placed on the Test circle and the Control circle and mixed thoroughly with the latex with the aid of the provided sterile plastic mixing stick. The mixing was done for three minutes and observed for agglutination under normal lighting conditions. The results of the Test and Control reactions were recorded before disposing the reaction card safely into disinfectant

\section{Determination of $\mathrm{MIC}$ and $\mathrm{MBC}$ of the fractions on MRSA clinical isolates}

Dilution schemes using formula $\mathrm{C}_{1} \mathrm{~V}_{1}=\mathrm{C}_{2} \mathrm{~V}_{2}$ are given as shown in table 1

$\mathrm{C}_{\mathrm{F} 1}=$ Stock concentration of the fractions (EAF \& $\mathrm{DMF})=50 \mathrm{mg} / \mathrm{ml}$

$\mathrm{V}_{\mathrm{F} 1}=$ Volume of the fractions (EAF \& DMF) in the agar dilution $=$ to be determined

$\mathrm{C}_{\mathrm{F} 2}=$ Concentration of the fractions(EAF \& DMF) in agar dilution $(1 \mathrm{mg} / \mathrm{ml}-10 \mathrm{mg} / \mathrm{ml})$

$\mathrm{V}_{\mathrm{F} 2}=$ Volume of reaction mixture in MHA plate $=20$ $\mathrm{ml}$ 
International Journal of Trend in Scientific Research and Development (IJTSRD) ISSN: 2456-6470

Table 1: GC-MS determination of bioactive components the fractions

\begin{tabular}{|l|l|l|l|l|l|}
\hline $\mathbf{S} / \mathbf{N}$ & $\mathbf{C}_{\mathrm{F} 1}(\mathrm{mg} / \mathrm{ml})$ & $\mathbf{V}_{\mathrm{F} 1}(\mathrm{ml})$ & $\begin{array}{l}\mathrm{C}_{\mathrm{F} 2}(\mathrm{Conc} \text { of } \\
\mathbf{E A F} \& \text { DMIF) } \\
(\mathrm{mg} / \mathrm{ml})\end{array}$ & $\begin{array}{l}\text { Volume of } \\
\text { MHA }(\mathrm{ml})\end{array}$ & $\begin{array}{l}\mathbf{V}_{\mathrm{F} 2}(\mathrm{ml}) \\
\text { Volume of reaction } \\
\text { mixture }\end{array}$ \\
\hline 1 & 50 & 4.00 & 10 & 16.00 & 20 \\
\hline 2 & 50 & 3.60 & 9 & 16.40 & 20 \\
\hline 3 & 50 & 3.20 & 8 & 16.80 & 20 \\
\hline 4 & 50 & 2.80 & 7 & 17.20 & 20 \\
\hline 5 & 50 & 2.40 & 6 & 17.60 & 20 \\
\hline 6 & 50 & 2.00 & 5 & 18.00 & 20 \\
\hline 7 & 50 & 1.60 & 4 & 18.40 & 20 \\
\hline 8 & 50 & 1.20 & 3 & 18.80 & 20 \\
\hline 9 & 50 & 0.80 & 2 & 19.20 & 20 \\
\hline 10 & 50 & 0.40 & 1 & 19.60 & 20 \\
\hline
\end{tabular}

Gas chromatography-mass spectrometry was performed on the two fractions of the Moringa oleifera root bark

\section{Statistical Analysis}

Results were expressed as mean $\pm \mathrm{SD}$ and differences between sets obtained were determined using ANOVA followed by Duncan post Hoc Test with the use of SPSS v 17 software. Differences were considered significant at $\mathrm{p}<0.05$.

\section{RESULTS}

Table 2: Percentage yield of the Methanol crude extract and Fractions

\begin{tabular}{|l|l|l|}
\hline $\begin{array}{l}\text { Initial weight of ground moringa } \\
\text { root bark } 3000 \mathrm{~g}\end{array}$ & Final weight of extract $(\mathrm{g})$ & Percentage yield of extract \\
\hline Methanol crude extract & $850.60 \pm 20.80$ & $28.35 \%$ \\
\hline \multicolumn{2}{|l|}{ Percentage yield of the fractions from Methanol crude extract } \\
\hline Ethyl acetate fraction & $50.90 \pm 8.70$ & $5.98 \%$ \\
\hline Dichloromethane & $150.70 \pm 9.50$ & $17.71 \%$ \\
\hline
\end{tabular}

\section{Qualitative Phytochemical analysis of the fractions of the extract}

Table 3: Results of Phytochemical Analysis of the Methanol Extracts and Fractions

\begin{tabular}{|c|c|c|c|c|}
\hline $\begin{array}{l}\text { Chemical } \\
\text { Constituent }\end{array}$ & Test & $\begin{array}{l}\text { Crude methanol } \\
\text { extract } \\
\text { (MIE) }\end{array}$ & $\begin{array}{l}\text { Dichlorometha } \\
\text { ne fraction } \\
\text { (DMF) } \\
\end{array}$ & $\begin{array}{l}\text { Ethyl acetate } \\
\text { fraction (EF) }\end{array}$ \\
\hline Alkaloids & $\begin{array}{l}\text { Dragendorff's reagent } \\
\text { Mayer's reagent } \\
\text { Wagner's reagent }\end{array}$ & ++++ & + & - \\
\hline Glycosides & Fehling's solution I and II & ++ & - & + \\
\hline Steroids & General Test & ++ & ++ & + \\
\hline Terpenoids & General Test & + & + & + \\
\hline Flavonoids & $\begin{array}{l}\text { Ammonium Test } \\
1 \% \text { Aluminium Chloride } \\
\text { solution Test. }\end{array}$ & ++ & + & ++++ \\
\hline Saponins & Frothing Test & ++ & - & \\
\hline
\end{tabular}


International Journal of Trend in Scientific Research and Development (IJTSRD) ISSN: 2456-6470

\begin{tabular}{|l|l|l|l|l|}
\hline & $\begin{array}{l}\text { Emulsion Test } \\
\text { Fehling's Test }\end{array}$ & & \\
\hline Tannins & $\begin{array}{l}\text { Ferric chloride Test } \\
\text { Lead Acetate Test }\end{array}$ & ++ & - & - \\
\hline Resins & $\begin{array}{l}\text { Precipitation Test } \\
\text { Colour Test }\end{array}$ & ++ & ++++ & - \\
\hline Reducing Sugar & Fehling's solution I and II & ++ & - & - \\
\hline Proteins & $\begin{array}{l}\text { Millon's Test } \\
\text { Xanthoproteic Reaction } \\
\text { Test } \\
\text { Picric Acid Test } \\
\text { Biuret Test }\end{array}$ & +++ & & - \\
\hline Fats and Oil & General filter paper Test & ++ & & \\
\hline Carbohydrate & Molisch's & +++ & ++ & - \\
\hline
\end{tabular}

Key:

$(-)$ : Not present.

$(+)$ : Present in small concentration.

$(++)$ : Present in moderately high concentration.

$(+++)$ : Present in very high concentration.

$(++++)$ : Abundantly presently.

Table 4: MIC and MBC of ethyl acetate fraction in $\mathrm{mg} / \mathrm{ml}$

\begin{tabular}{|c|c|c|c|c|c|c|c|}
\hline $\mathbf{S} / \mathbf{N}$ & $\begin{array}{l}\text { Clinical } \\
\text { isolates }\end{array}$ & $\mathrm{MIC}$ & $\mathrm{MBC}$ & $\mathrm{S} / \mathbf{N}$ & $\begin{array}{l}\text { Clinical } \\
\text { isolates }\end{array}$ & MIC & $\mathrm{MIBC}$ \\
\hline 1 & SP4 & $5.6 \pm 0.5$ & $6.3 \pm 0.5$ & 21 GाEाtा & EN390 & $4.6 \pm 0.5$ & $5.3 \pm 1.1$ \\
\hline 2 & SS8 & $4.3 \pm 1.1$ & $5.6 \pm 1.1 \mathrm{e}$ & 22 and & SS310 & $5.3 \pm 0.3$ & $6.6 \pm 0.5$ \\
\hline 3 & AB20 & $4.6 \pm 1.5$ & $6.6 \pm 0.5$ & 23 & OW417 & $4.6 \pm 0.3$ & $5.3 \pm 0.3$ \\
\hline 4 & SP22 & $5.3 \pm 0.5$ & $6.3 \pm 0.5$ & 24 & $\mathrm{AB} 570$ & $4.8 \pm 0.2$ & $5.6 \pm 0.5$ \\
\hline 5 & OW30 & $5.0 \pm 0.5$ & $6.6 \pm 0.5$ & 25 & OW578 & $5.3 \pm 0.5$ & $6.5 \pm 1.0$ \\
\hline 6 & SS33 & $5.3 \pm 0.3$ & $6.3 \pm 1.0$ & $26^{-0410}$ & AB600 & $5.3 \pm 1.1$ & $5.3 \pm 1.0$ \\
\hline 7 & EN35 & $5.6 \pm 0.5$ & $7.3 \pm 0.5$ & 27 & OW620 & $4.5 \pm 0.5$ & $5.0 \pm 0.5$ \\
\hline 8 & OW36 & $5.0 \pm 0.5$ & $6.3 \pm 0.5$ & 28 & SP651 & $5.5 \pm 0.5$ & $6.6 \pm 0.5$ \\
\hline 9 & EN38 & $5.3 \pm 0.5$ & $6.6 \pm 0.3$ & 72 & OW819 & $3.6 \pm 0.3$ & $4.3 \pm 0.5$ \\
\hline 10 & SS42 & $5.6 \pm 0.3$ & $6.3 \pm 0.5$ & 30 & EN831 & $4.3 \pm 0.3$ & $4.6 \pm 1.0$ \\
\hline 11 & OW53 & $6.6 \pm 0.5$ & $7.3 \pm 0.5$ & $31 \longrightarrow$ & AB841 & $5.6 \pm 0.5$ & $6.6 \pm 0.5$ \\
\hline 12 & SS57 & $5.3 \pm 0.5$ & $6.6 \pm 1.0$ & 32 & OW940 & $4.3 \pm 0.5$ & $5.6 \pm 0.3$ \\
\hline 13 & AB61 & $6.6 \pm 0.3$ & $7.3 \pm 0.5$ & 33 & OW947 & $3.5 \pm 0.3$ & $5.3 \pm 0.3$ \\
\hline 14 & EN62 & $5.6 \pm 0.5$ & $6.6 \pm 0.3$ & 34 & AB1009 & $4.5 \pm 0.3$ & $5.6 \pm 1.0$ \\
\hline 15 & OW123 & $5.0 \pm 0.5$ & $6.3 \pm 1.0$ & 35 & OW1104 & $4.3 \pm 0.5$ & $5.6 \pm 0.5$ \\
\hline 16 & EN127 & $6.6 \pm 0.3$ & $7.5 \pm 0.5$ & 36 & SP1172 & $5.6 \pm 0.3$ & $7.3 \pm 1.0$ \\
\hline 17 & OW154 & $5.6 \pm 0.5$ & $6.5 \pm 0.5$ & 37 & OW1420 & $5.6 \pm 0.5$ & $6.3 \pm 0.3$ \\
\hline 18 & AB187 & $6.6 \pm 0.3$ & $7.2 \pm 1.1$ & 38 & OW1827 & $5.3 \pm 0.3$ & $6.0 \pm 0.5$ \\
\hline 19 & EN208 & $5.3 \pm 0.5$ & $6.5 \pm 0.5$ & 39 & AB1956 & $4.6 \pm 0.5$ & $5.6 \pm 1.1$ \\
\hline 20 & SS235 & $5.6 \pm 0.3$ & $6.3 \pm 0.5$ & & & & \\
\hline
\end{tabular}

Values were expressed as Mean $\pm \mathrm{SD}, \mathrm{N}=3$

Key:

SP: Sputum 
International Journal of Trend in Scientific Research and Development (IJTSRD) ISSN: 2456-6470

SS: Skin swab

AB: Abscess

OW: Open wound

EN: Ear/Nasal

Table 5: MIC and MBC of Dichloromethane fraction in $\mathrm{mg} / \mathrm{ml}$

\begin{tabular}{|c|c|c|c|c|c|c|c|}
\hline $\mathbf{S} / \mathbf{N}$ & $\begin{array}{l}\text { Clinical } \\
\text { isolates }\end{array}$ & MIC & $\mathrm{MIBC}$ & $\mathbf{S} / \mathbf{N}$ & $\begin{array}{l}\text { Clinical } \\
\text { isolates }\end{array}$ & $\mathrm{MIIC}$ & $\mathrm{MIBC}$ \\
\hline 1 & SP4 & $7.5 \pm 0.5$ & $8.7 \pm 0.5$ & 21 & EN390 & $7.5 \pm 0.5$ & $8.6 \pm 1.0$ \\
\hline 2 & SS8 & $6.3 \pm 1.1$ & $7.5 \pm 1.1$ & 22 & SS310 & $8.7 \pm 1.1$ & $9.5 \pm 1.3$ \\
\hline 3 & $\mathrm{AB} 20$ & $6.5 \pm 1.5$ & $7.8 \pm 0.5$ & 23 & OW417 & $8.3 \pm 0.5$ & $9.6 \pm 1.0$ \\
\hline 4 & SP22 & $8.2 \pm 0.5$ & $8.2 \pm 0.5$ & 24 & $\mathrm{AB} 570$ & $8.7 \pm 1.1$ & $8.8 \pm 0.5$ \\
\hline 5 & OW30 & $7.5 \pm 1.5$ & $9.2 \pm 0.5$ & 25 & OW578 & $6.7 \pm 0.5$ & $8.5 \pm 1.1$ \\
\hline 6 & SS33 & $7.6 \pm 0.5$ & $8.6 \pm 1.0$ & 26 & AB600 & $7.5 \pm 1.1$ & $8.7 \pm 1.0$ \\
\hline 7 & EN35 & $6.6 \pm 1.1$ & $7.8 \pm 0.5$ & 27 & OW620 & $8.5 \pm 1.5$ & $8.8 \pm 0.5$ \\
\hline 8 & OW36 & $8.7 \pm 1.1$ & $8.8 \pm 0.5$ & 28 & SP651 & $7.6 \pm 0.5$ & $8.8 \pm 0.5$ \\
\hline 9 & EN38 & $6.7 \pm 0.5$ & $7.8 \pm 1.1$ & 29 & OW819 & $6.2 \pm 1.1$ & $7.6 \pm 0.5$ \\
\hline 10 & SS42 & $7.5 \pm 1.0$ & $8.7 \pm 0.5$ & 30 & EN831 & $8.5 \pm 0.5$ & $9.5 \pm 1.1$ \\
\hline 11 & OW53 & $8.2 \pm 0.5$ & $9.5 \pm 0.5$ & 31 & $\mathrm{AB} 841$ & $9.3 \pm 0.5$ & $9.5 \pm 1.1$ \\
\hline 12 & SS57 & $7.8 \pm 0.5$ & $8.8 \pm 1.0$ & 32 & OW940 & $8.5 \pm 0.5$ & $9.7 \pm 1.1$ \\
\hline 13 & AB61 & $8.8 \pm 0.5$ & $9.5 \pm 0.5$ & 33 & OW947 & $7.8 \pm 1.1$ & $8.5 \pm 1.1$ \\
\hline 14 & EN62 & $8.7 \pm 0.5$ & $9.6 \pm 1.1$ & 34 vण & AB1009 & $7.5 \pm 1.1$ & $7.6 \pm 1.1$ \\
\hline 15 & OW123 & $7.8 \pm 0.5$ & $8.8 \pm 1.1$ & 35 cient & OW1104 & $8.0 \pm 0.5$ & $8.5 \pm 0.5$ \\
\hline 16 & EN127 & $8.5 \pm 0.5$ & $9.5 \pm 0.5$ & 36 & SP1172 & $8.5 \pm 1.1$ & $9.6 \pm 1.0$ \\
\hline 17 & OW154 & $7.8 \pm 0.5$ & $8.3 \pm 0.5$ & 37 & OW1420 & $8.7 \pm 0.5$ & $8.8 \pm 0.5$ \\
\hline 18 & AB187 & $8.5 \pm 0.5$ & $9.7 \pm 1.1 \mathrm{e}$ & 38 nent & OW1827 & $9.3 \pm 1.1$ & $9.5 \pm 0.5$ \\
\hline 19 & EN208 & $7.8 \pm 0.5$ & $8.7 \pm 0.5$ & 39 & $\mathrm{AB} 1956$ & $8.2 \pm 0.5$ & $9.6 \pm 1.1$ \\
\hline 20 & $\mathrm{SS} 235$ & $8.8 \pm 0.5$ & $9.3 \pm 0.5$ & $56=6470$ & 10 & 3 & \\
\hline
\end{tabular}

Values were expressed as Mean $\pm \mathrm{SD}, \mathrm{N}=3$

Key:

SP: Sputum

SS: Skin swab

AB: Abscess

OW: Open wound

EN: Ear/Nasal

Table 6: GC-MS report of ethyl acetate fraction

\begin{tabular}{|l|l|l|l|}
\hline \multicolumn{1}{|c|}{ Structural analogue } & Rt (min) & Area \% & Peak \\
\hline Stigmast-4-en-3-one, Cholest-4-en-26-oic acid, 3-oxo-Cholest-4-en-3-one & 46.49 & 8.72 & 1 \\
\hline $\begin{array}{l}\text { 5-Bromovaleric acid, 2,6-dimethylnon-1-en-3-yn-5-yl ester , Cyclobuta } \\
\text { [1,2:3,4]dicyclooctene-1,7(2H,6bH)-dione. }\end{array}$ & 46.58 & 3.19 & 2 \\
\hline $\begin{array}{l}\text { 3-Chloropropionic acid, 2,6-dimethylnon-1-en-3-yn-5-yl ester, 2-Amino-4- } \\
\text { methyl-3-pyridinol, Pregn-4-en-3-one. }\end{array}$ & 46.62 & 5.80 & 3 \\
\hline $\begin{array}{l}\text { 4,22-Stigmastadiene-3-one, 24S--Ethyl-5.alpha-cholesta-2-dien-6-one, } \\
\text { Spinasterone }\end{array}$ & 48.54 & 12.29 & 4 \\
\hline $\begin{array}{l}\text { 4,22-Stigmastadiene-3-one, 4,22-Cholestadien-3-one, } \\
\text { Ergosta-4,22-dien-3-one }\end{array}$ & 48.62 & 10.73 & 5 \\
\hline
\end{tabular}




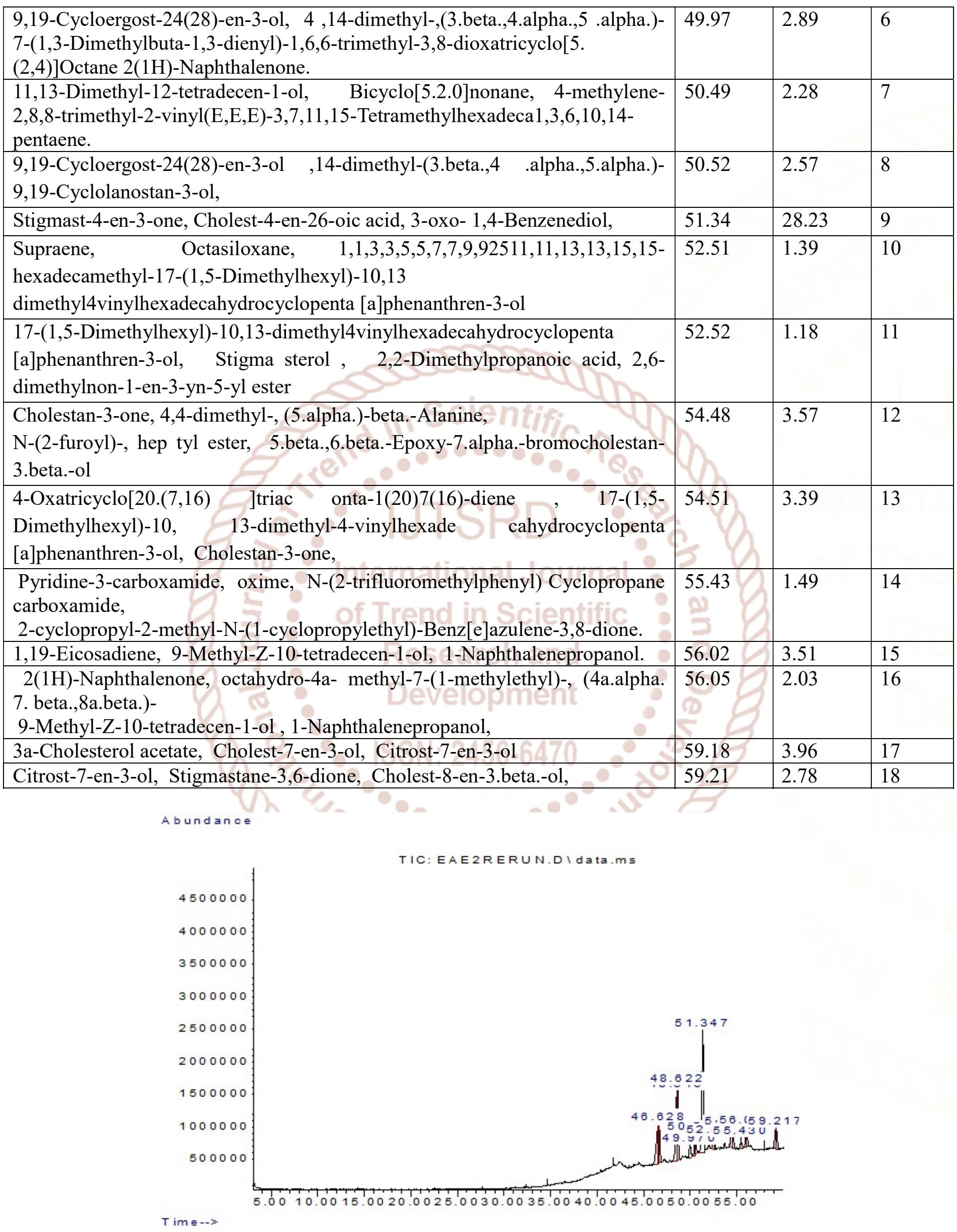

Fig. 1: MS fragment of ethyl acetate fraction composition 
International Journal of Trend in Scientific Research and Development (IJTSRD) ISSN: 2456-6470

Fragmentation pattern for the identified compounds are presented in Fig. 1. The abundance of each compound, the peak height, percentage area and retention time are all shown in the graph. There is overlapping of fragments, showing that some of the functional groups can be repeated in another peak.

Table 7: GC-MS report of dichloromethane fraction

\begin{tabular}{|c|c|c|c|}
\hline Structural analogue & $\operatorname{Rt}(\min )$ & Area $\%$ & Peak \\
\hline Benzylamine & 11.33 & 0.46 & 1 \\
\hline Benzyl isocyanate, Phthalimidin & 16.87 & 1.96 & 2 \\
\hline 3-Methyl-4-isopropylphenol, Thymol , 2-methyl-5-(1-methylethyl) Phenol & 25.97 & 1.57 & 3 \\
\hline N-Benzylformamide & 30.21 & 0.68 & 4 \\
\hline $\begin{array}{l}\text { Acetamide, N-(phenyl methyl)- 2-Methyl-5-butylpyridine , Dimethyl (1E)-N- } \\
\text { hydroxyethanimidoy lphosphonate }\end{array}$ & 32.09 & 2.78 & 5 \\
\hline Humulene & 32.35 & 0.56 & 6 \\
\hline $\begin{array}{l}\text { 1,6-Cyclodecadiene, 1-methyl-5-methylene-8-(1-methylethyl)-1H } \\
\text { Cyclopenta[1,3]cyclopropa[1,2]benzene, octahydro-7-methyl-3-methylene-4- } \\
\text { (1-methylethyl)-[3aS-(3a alpha.,3b.beta.,4.beta.,7.alpha.,7 aS*)]-.beta.- } \\
\text { copaene }\end{array}$ & 33.57 & 0.77 & 7 \\
\hline $\begin{array}{l}1, \quad 2, \quad 3, \quad 5, \quad 6, \quad 8 \text { a-hexahydr-4,7-dimethyl-1-(1-methylethyl)-,(1S-cis)- } \\
\text { Naphthalene. }\end{array}$ & 35.41 & 1.06 & 8 \\
\hline tau.-Muurolol, alpha-Cadinol, cis-muurola-3,5-diene & 40.21 & 0.99 & 9 \\
\hline $\begin{array}{l}\text { alpha-Cadinol, Cyclohexane, 1-ethenyl-1-methyl-2- (1-methylethenyl)-4-(1- } \\
\text { methylethylidene)-Epiglobulol }\end{array}$ & 40.71 & 1.70 & 10 \\
\hline Hexadecanoic acid, Pentadecanoic acid, 14-methyl-,methyl ester & 50.64 & 5.89 & 11 \\
\hline n-Hexadecanoic acid & 52.16 & 1.42 & 12 \\
\hline $\begin{array}{l}\text { Cyclopropaneoctanoic acid, 2- methyl ester 9-Octadecenoic acid, (Z)-methyl } \\
\text { Ster,cis-10-Heptadecenoic acid. }\end{array}$ & 53.39 & 0.67 & 13 \\
\hline Heptadecanoic acid, Hexadecanoic acid. Research and & 53.92 & 0.39 & 14 \\
\hline 9,12-Octadecadienoic acid, 10,13-Octadecadienoic acid, & 56.07 & 4.29 & 15 \\
\hline 9-Octadecenoic acid, 8-Octadecenoic acid, methyl ester & 56.26 & 3.32 & 16 \\
\hline 9-Octadecenoic acid, 11-Octadecenoic acid, & 56.44 & 0.96 & 17 \\
\hline \begin{tabular}{|l|l|} 
Methyl stearate & $\mathrm{SSN}_{2}: 2456=6470$ \\
\end{tabular} & 57.11 & 1.28 & 18 \\
\hline $\begin{array}{l}\text { Urea, N,N'-bis(phenyl methyl)-Benzeneacetamide, alpha.-amino-Benzene } \\
\text { acetic acid, }\end{array}$ & 64.34 & 1.87 & 19 \\
\hline Docosanoic acid, methyl ester, Tricosanoic acid, methyl ester & 68.62 & 0.53 & 20 \\
\hline Bis(2-ethylhexyl) phthalate, Di-n-octyl phthalate & 21.69 & 0.37 & 21 \\
\hline $\begin{array}{l}\text { 5-Fluoro-1,3-bis[phenylmethyl]-2,4 }(1 \mathrm{H}, 3 \mathrm{H}) \text {-pyrimidinedione, } \\
\text { 5-Benzyloxy-6-methoxy-8-nitroquinoline, Ethisterone }\end{array}$ & 70.47 & 0.39 & 22 \\
\hline $\begin{array}{l}\text { 2-Thiazolamine, 4-(4-methoxyphenyl )-N-(4-methylphenyl)-Naphtho[1,8- } \\
\text { cd]-(1,2,6)-phosphadiazine, 2-phenyl-2-thioxo-1,3(2H)-di hydro-1- } \\
\text { Dimethyl(ethenyl)silyloxy-3-phenylpropane }\end{array}$ & 23.71 & 1.20 & 23 \\
\hline $\begin{array}{l}\text { Benzene, 1-isocyanato-3-methoxy- 1H-S-Triazolo[1,5-a]pyridin-4-ium, 2- } \\
\text { hydroxy-1-methyl-, hydroxide, inner salt, Didodecylphthalate, }\end{array}$ & 76.72 & 0.73 & 24 \\
\hline $\begin{array}{l}\text { Benzonitrile, 2-fluoro-4-(4'-propyl 1[1,1'-bicyclohexyl]-4-yl)-Oxazole, 2-(3- } \\
\text { methoxyphenyl)-5-phe nyl-Cyclopropane, carboxamide, }\end{array}$ & 79.02 & 0.39 & 25 \\
\hline $\mathrm{N}$-(phenylmethyl)- Acetamide, & 80.83 & 0.69 & 26 \\
\hline $\begin{array}{l}\text { Cedran-diol, }(8 \mathrm{~S}, 14)-\text { Phthalic acid, hexyl 1-phenylpropy } 1 \text { este, } \\
\text { Benzo[1,3]dioxole-5-carboxylic aci d (5-chloro-2-oxo-1,2-dihydro-indo 1-3- } \\
\text { ylidene)-hydrazide }\end{array}$ & 81.51 & 0.51 & 27 \\
\hline Stigmastan-3,5-diene; .beta.-Sitosterol acetate & 81.97 & 0.57 & 28 \\
\hline Campesterol, (3.beta.)-Ergost-7-en-3-ol, & 85.32 & 0.92 & 29 \\
\hline Stigmasterol & 86.28 & 1.13 & 30 \\
\hline
\end{tabular}


Continuation ofGC-MS report of dichloromethane fraction

\begin{tabular}{|c|c|c|c|}
\hline Structural analogue & $\operatorname{Rt}(\min )$ & Area \% & Peak \\
\hline $\begin{array}{l}\text { Pyridine-3-carboxamide, oxime, N-( 2-trifluoromethylphenyl)-3-n-Heptyl-7-methyl-9-(2,6, } \\
\text { thylcyclohex-1-enyl)nona-2,4,6,8- etraenal ,1,3-Dioxolane. }\end{array}$ & 87.33 & 0.38 & 31 \\
\hline $\begin{array}{l}\text { 4,4,6a,6b,8a,11,12,14b-Octamethyl-octadecahydro-2H-picen-3-one, } 2(1 \mathrm{H}) \text { Naphthalenone, } \\
3,5,6,7,8,8 \mathrm{a} \text {-hexahydro-4,8a-dimethyl-6-(1-methylethenyl)-gamma.-Sitosterol. }\end{array}$ & 88.06 & 1.63 & 32 \\
\hline $\begin{array}{l}\text { 5-Bromovaleric acid, 2,6-dimethylnon-1-en-3-yn-5-yl ester, } \\
\text { 6-Bromohexanoic acid,4-methoxyphenyl ester Cyclohexanecarboxylic } \\
\text { methoxyphenyl ester }\end{array}$ & 10.14 & 89.57 & 33 \\
\hline 4,22-Stigmastadiene-3-one, Ethyl-5.alpha.-cholesta -dien-6-one, Ergosta-4,22-dien-3-one & 90.75 & 11.91 & 34 \\
\hline Cholest-7-en-3-one, 4, 4-dimethyl-(5.alpha.)- Stigmasterol, Ergost-25-ene-3,5,6,12-tetrol & 91.10 & 0.44 & 35 \\
\hline $\begin{array}{l}\text { Benzofran-3-one, 2-[3,4-dihydroxybenzylidene]-6-hydroxy- Stigmasterol, Ergosta-4,6,22- } \\
\text { trien-3-one }\end{array}$ & 91.70 & 1.60 & 36 \\
\hline $\begin{array}{l}\text { C(14a)-Homo-27-nor-14.beta.-gammaceran-3.alpha.-ol, } 17-(1,5 \text {-Dimethylhexyl)-10,13- } \\
\text { dimet hyl-4 vinylhexadecahydrocyclopenta[a]phenanthren-3-ol, } \\
\text { 9,19-Cyclo-25,26-epoxyergostan-3-o 1, 4,4,14-trimethyl-, acetate }\end{array}$ & 92.21 & 2.77 & 37 \\
\hline Stigmast-4-en-3-one, Testosterone, Androst-4-en-3-one, & 93.05 & 12.33 & 38 \\
\hline $\begin{array}{l}\text { Nickel, cyclopentadienyl-(dicycloh exylphosphino)benzyl-o-yl- } \\
\text { 3.alpha.-ol, Stigmasta-4,6,22-trien-3.beta.-ol }\end{array}$ & 94.09 & 1.53 & 39 \\
\hline -4,6,22-trien-3.beta.-ol , Stigmasta-4,6,22-trien-3.alpha.-ol, Stigmasta-3,5-dien-7-one & 94.11 & 1.02 & 40 \\
\hline $\begin{array}{l}\text { Cholestan-3-one, 4,4-dimethyl-, (alpha.)-Stigmastan-7-one, } \\
17 \text { (1,5Dimethylhexyl)-10,13-dimethyl-4vinylhexadecahydrocyclopenta[a]phenanthren-3-ol }\end{array}$ & 96.05 & 2.17 & 41 \\
\hline Cholestan-3-one, 4,4-dimethyl-Dihydrosarsasapogenin-5,17(20)-die Phenol & 96.08 & 1.98 & 42 \\
\hline $\begin{array}{l}\text { C(14a)-Homo-27-nor-14.beta--gammaceran-3.alpha.-ol } \\
\text { 22-Stigmasten-3-one ,4-Diethyl thiophosphoryl-3-thiometh } 1 \text { yl allophanate }\end{array}$ & 97.57 & 3.62 & 43 \\
\hline $\begin{array}{l}\text { Stigmastane-3,6-dione, (5.alpha.)- } \begin{array}{c}\text { Lanostane, 11,18-epoxy-, (11.beta. 17-(1,5- } \\
\text { Dimethylhexyl)-2-(1-hydrox } \\
\text { ahydrocyclopenta[a]phenanthren-3-one }\end{array} \text { Interly yethylidene)-10,13-dimethylhexadec } \\
\end{array}$ & 100.66 & 1.80 & 44 \\
\hline $\begin{array}{l}\text { Stigmastane-3,6-dione } \\
\text { Diazatetrabenzo[a,cd,j,lm] per ylene }\end{array}$ & 100.71 & 1.62 & 45 \\
\hline $\begin{array}{l}\text { Benzenepropanoic acid, 3,5-bis(1,1 -dimethylethyl)-4-hydroxy-, octade Benzenepropanoic } \\
\text { acid, -dimethylethyl)-4-hydroxy-,octade cyl ester, beta.-Tocopherol, }\end{array}$ & 101.15 & 2.59 & 46 \\
\hline Benzenepropanoic acid, 3,5-bis(1,1 -dimethylethyl)-4-hydroxy-, octade cyl ester & 101.19 & 2.43 & 47 \\
\hline
\end{tabular}

\section{Abundarce}

TIC CE3Ddatams

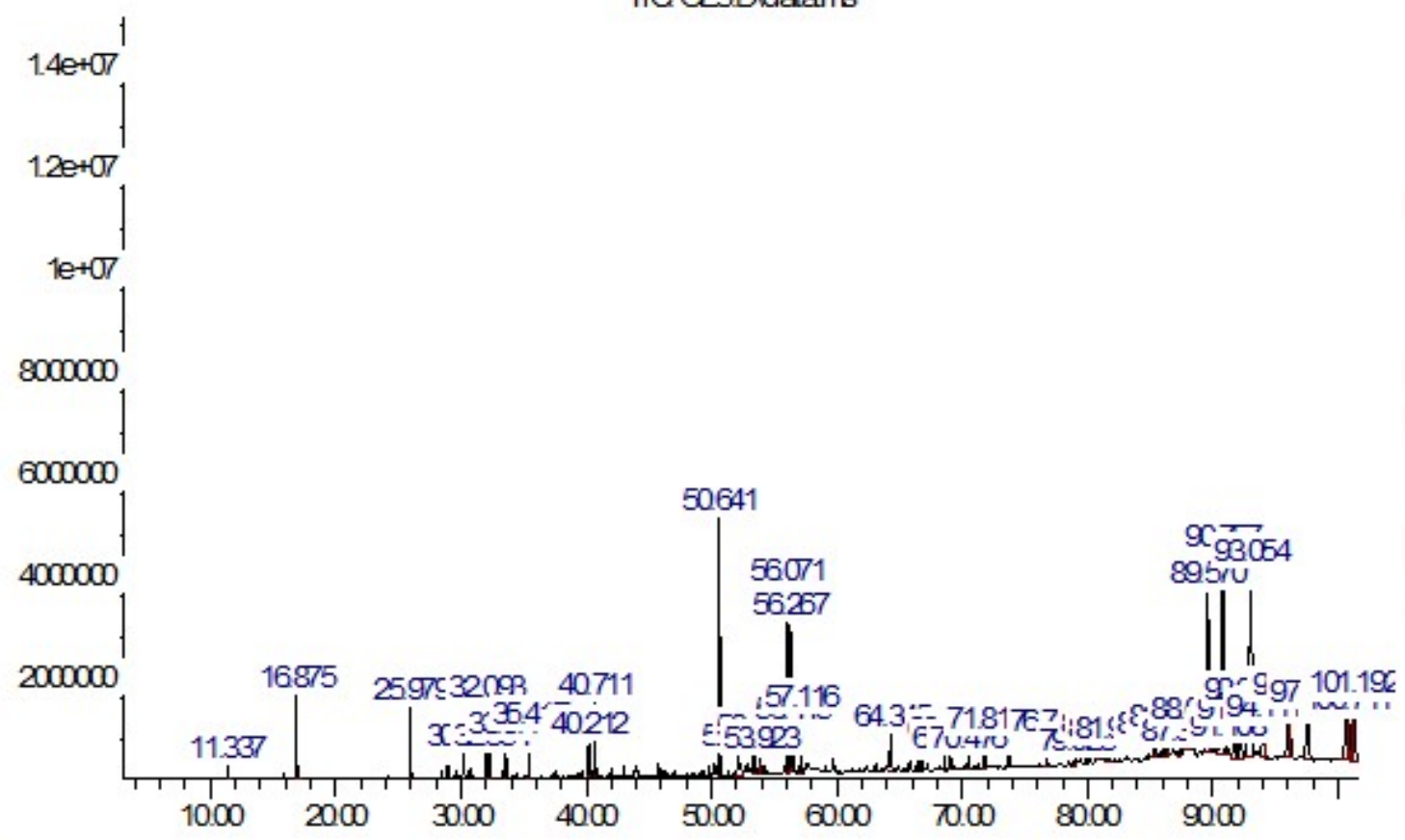

Time $\rightarrow$

Fig. 2: MS fragment of dichloromethane fraction composition 
Fragmentation pattern for the identified compounds are presented in Fig. 2.

The abundance of each compound, the peak height, percentage areas and retention time are all shown in the graph. There is also an overlapping of fragments, showing that some of the functional groups are repeated in another peak.

\section{Discussion}

\section{Percentage yield of extractand fractions}

The extractive values are useful to evaluate the chemical constituents present in the crude drug and also help in estimation of specific constituents soluble in a particular solvent [24] (Ozarkar) [25]. The percentage yield of the extract and fractions indicates a good yield. The methanol crude extract $850.60 \pm$ $20.8 \mathrm{~g}(28.35 \%)$. The fact that dichloromethane fraction yield is more thanethyl acetate fraction means there are more polar phytochemical constituents in the roots of Moringa oleifera than non-polar [25, 26]

\section{Qualitative phytochemical analysis of methanol extract and fractions}

Phytochemical compounds may inhibit bacterial growth by mechanisms different from presently used treatment regimens, and could therefore be of clinical value in the treatment of resistant bacteria, including MRSA. Tannins, flavonoids, alkaloids, essential oils and many phenolic compounds serve as plant defense mechanisms against predation by insects, herbivores and infection by microorganisms (Cowan) [120].

\section{MIC and MBC of the fractions}

The results of the antibacterial testing of the fractions against all the MRSA clinical isolate as display in table 12 and 13, revealed that ethyl acetate fraction (EF) showed better activity than dichloromethane (DMF) fraction, possibly because of the presence of high concentration of flavonoids and other secondary metabolites eluted by the solvent, which has been shown to enhance antimicrobial property of a plant [13-15]. The MRSA isolates for this study restricted all the popular antibiotics used in the hospitals in varying degrees, but was inhibited by ethyl acetate fraction at concentrations of $3.5 \mathrm{mg} / \mathrm{ml}$ as the least MIC, $4.3 \mathrm{mg} / \mathrm{ml}$ as the least MBC and $6.6 \mathrm{mg} / \mathrm{ml}$ and $7.5 \mathrm{mg} / \mathrm{ml}$ as the highest MIC and $\mathrm{MBC}$, while dichloromethane fraction (DMF) . This confirms the presence of bioactive principles in both fractions but the most potent component against the strain of MRSA is found in ethyl acetate fraction which can be formulated for the treatment of infections by the MRSA isolates.

\section{Gas chromatography-mass spectrometry (GC-MS) of the fractions}

A large numbers of compounds were identified in the fractions with these three compounds having the highest percentages, Stigmast-4-en-3-one (36.95\%), molecular formula $\mathrm{C}_{29} \mathrm{H}_{48} \mathrm{O}$, M.W 412, a ketone steroid with antimicrobial activities, antioxidant, antiinflammatory, antiarthritic, antiasthma and diuretic activities (S. John and P. Kumar) [355], (Jennings and shibamoto)[357], Cholest-4-en-26-oic acid (36.95\%) Molecular formula $\mathrm{C}_{27} \mathrm{H}_{42} \mathrm{O}_{4}$, M.W 430, Aliphatic acid sesterterpenes that regulates the metabolism of cholesterol and homeostasis [356] , 3-oxo- 1,4Benzenediol (28.23\%) , $\mathrm{C}_{18} \mathrm{H}_{18} \mathrm{~N}_{2} \mathrm{O}_{5}, \quad$ M.W 342, Oxygenated aldehyde with antimicrobial and antioxidant properties, antimycobacterial activity [353]. Other significant constituents with bioactivities are Spinasterone $12.29 \%$, a seroid compound with antibacterial and antifungal activities [354], 5Bromovaleric acid 3.19\%, 2-Amino-4-methyl-3pyridinol $5.8 \%$, Ergosta-4,22-dien-3-one 10.73\%, Pregn-4-en-3-one $\quad 5.8 \%, \quad 2(1 \mathrm{H})$-Naphthalenone $2.89 \%$, Pentaene $2.28 \%$, (5.alpha.)-beta.-Alanine $3.57 \%$, Supraene $1.39 \%$, Oxime $1.49 \%$ and Citrost-7en-3-ol 6.7\% [354,355, 356, 357, 358].

\section{CONCLUSION}

Moringa oleifera is already highly esteemed by people in the tropics and sub-tropics for the many ways it is used nutritionally by people and medicinally by local herbalist. In recent years, laboratory investigations have confirmed the efficacy of some of these applications as found in leaves, flowers, pods, roots, root bark and stem bark, gum, seeds and seed oil [116-120].Ethyl acetate (EAF) fraction is more potent than dichloromethane fraction; this indicates that the most active compound against the MRSA can be isolated from the EAF for further analysis.

\section{Conflict of Interest}

No conflict of interest 


\section{REFERENCES}

1. Dahot M. U. 1988. Vitamin contents of flowers and seeds of Moringa oleifera. Pak J Biochem 21: $1-24$.

2. Faizi S, Siddiqui B, Saleem R, Saddiqui S, Aftab K. 1994a. Isolation and structure elucidation of new nitrile and mustard oil glycosides from $M$. oleifera and their effect on blood pressure. J. Nat Prod 57: 1256-1261.

3. Mehta LK, Balaraman R, Amin AH, Bafna PA, Gulati OD. 2003. Effect of fruits of M. oleifera on the lipid profile of normal and hypercholesterolaemic rabbits. J. Ethnopharmacol 86: 191-195.

4. Gilani AH, Aftab K, Shaheen F et al. 1992. Antispasmodic activity of active principle from Moringa oleifera. In Nat. Drugs and Digest. Tract, Capasso F, Mascolo N [Eds]. EMSI: Rome, 60-63.

5. Dangi SY, Jolly CI, Narayana S. 2002. Antihypertensive activity of the total alkaloids from the leaves of M. oleifera. Pharm Biol 40: 144 148.

6. Gilani AH, Aftab K, Suria A et al. 1994a. Pharmacological studies on hypotensive and spasmodic activities of pure compounds from Moringa oleifera. Phytother Res 8: 87-91.

7. Pal SK, Mukherjee PK, Saha BP. 1995a. Studies on the antiulcer activity of Moringa oleifera leaf extract on gastric ulcer models in rats. Phytother Res 9: 463-465.

8. Eilert U, Wolters B, Nadrtedt A. 1981. The antibiotic principle of seeds of Moringa oleifera and Moringa stenopetala. Planta. Med 42: 55-61.

9. Bhatnagar SS, Santapau H, Desai JDH, Yellore S, Rao TNS 1961. Biological activity of Indian medicinal plants. Part 1. Antibacterial, antitubercular and antifungal action. Indian J Med Res 49: 799-805.

10. Miller RG, Kopfler FC, Kelty KC, Stober JA, Ulmer NS. 1984. The occurrence of aluminum in drinking water. J Am Water Works Assoc 76: 8491.

11. Obioma UN, Adikwu MU. 1997. Investigation on some physiochemical antioxidant and toxicological properties of Moringa oleifera seed oil. Acta Pharm 47: $287-290$.
12. Jahn SAA. 1984. Effectiveness of traditional flocculants asprimary coagulants and coagulant aids for the treatment of tropical waters with more than a thousand fold flocculation in turbidity. Water Supply 2: 8-10.

13. Okuda T, Baes AU, Nishijima W, Okada M. 1999. Improvement of extraction method of coagulation active components from Moringa oleifera seed. Water Res 33: 3373-3378.

14. Gassenschmidt U, Jany KD, Tauscher B, Niebergall H. 1995. Isolation and characterization of a flocculating protein from Moringa oleifera Lam. Biochim Biophys Acta 1243: 477- 481.

15. Muyibi SA, Evison LM. 1995a. Moringa oleifera seeds for softening hard water. Water Res 29: 1099-1104.

16. Broin M, Santaella C, Cuine S, Kokou K, Peltier G, Joet T. 2002. Flocculent activity of a recombinant protein from Moringa oleifera Lam. seeds. Appl Microbiol Biotechnol 60: 114-119.

17. Madsen M, Schlundt J, Omer El-FE. 1987. Effect of water coagulation by seeds of Moringa oleifera on bacterial concentration. J Trop Med Hyg 90: 101109

18. Olsen A. 1987. Low technology water purification by / bentonite clay and Moringa oleifera seed flocculation as performed in Sudanese villages: effects on Schistosoma mansoni cercariae. Water Res 21: $517-522$.

19. Casey TJ. 1997. Unit Treatment Processes in Water and Waste water Engineering. John Wiley \& Sons: London.

20. Silvestro L, Weiser JN, Axelsen PH. 2000. Antibacterial and antimembrane activities of cecropin A in Escherichia coli. Antimicrob Agents Chemother 44: 602-607.

21. Trease, G.E and Evans, W.C, (1996). Textbook of Pharmacognosy. $14^{\text {th }}$ Ed. W.B. Harborne, J.B.C. (1973). Phytochemical Methods, Chapman and Hall, London, p.279.

22. Iwu, M.M. (1978). Practical Pharmacognosy manual of Natural Products. Olowe O.A., Eniola K.I.T., Olowe R.A., Olayemi A.B, Antimicrobial Susceptibility and Beta-lactamase detection of MRSA in Osogbo. SW Nigeria, Nature and Science, 5(3),2007,

23. Ozarkar, K. R., 2005 Studies on anti-inflammatory effects of two herbs Cissus quadrangularis Linn. and Valeriana wallichi DC usingmousemodel. 
International Journal of Trend in Scientific Research and Development (IJTSRD) ISSN: 2456-6470

Ph.D. Thesis,University of Mumbai, 28. Prabhadevi V, Sahaya Sathish S, Johnson M, Mumbai.[Journal of applied Sciences Venkatramani B, Janakiraman Phytochemical studies on Allamanda cathartica L. using GC-MS, Asian Pacific Journal of Tropical Biomedicine (2012)S550-S554.

25. Gupta R, Kannan GM, Sharma M, Flora SJS. Therapeutic effects of Moringa oleifera on arsenicinduced toxicity in rats. Environmental Toxicology and Pharmacology 2005; 20(3): 456-464.Chuang PH, Lee CW, Chou JY, Murugan

26. Shieh M. BJ, Hueih-Minchen. Antifungal activity of crude extracts and essential oil of Moringa oleifera Lam. Bioresource Technology 2007; 98:232- 236.

27. Ahmed S. John, Kumar P., GC-MS Study of the Excoecaria agollocha Leaf extract from Pitchavaram, Tamil nadu, India, Researcher 2012;4(6):10-14]. (ISSN: 1553-9865). http://www.sciencepub.net/researcher. 3

29. Roberts M. Edible and Medicinal Flowers 2000; New Africa Books, Claremont. p. 52.

30. Sastri BN. The Wealth of India 1962; Council of Scientific and Industrial Research, New Delhi. p.425.

31. Adams 2007 r1, Release Guide, MSC.Software Corporation, 2 MacArthur Place, Santa Ana, CA 92707, USA. www.mscsoftware.com.

32. The NIST 14 Mass Spectral Library \& Search Software (NIST 2014/EPA/NIH) (previously NIST 2011/NIST11/NIST 2008/NIST08)

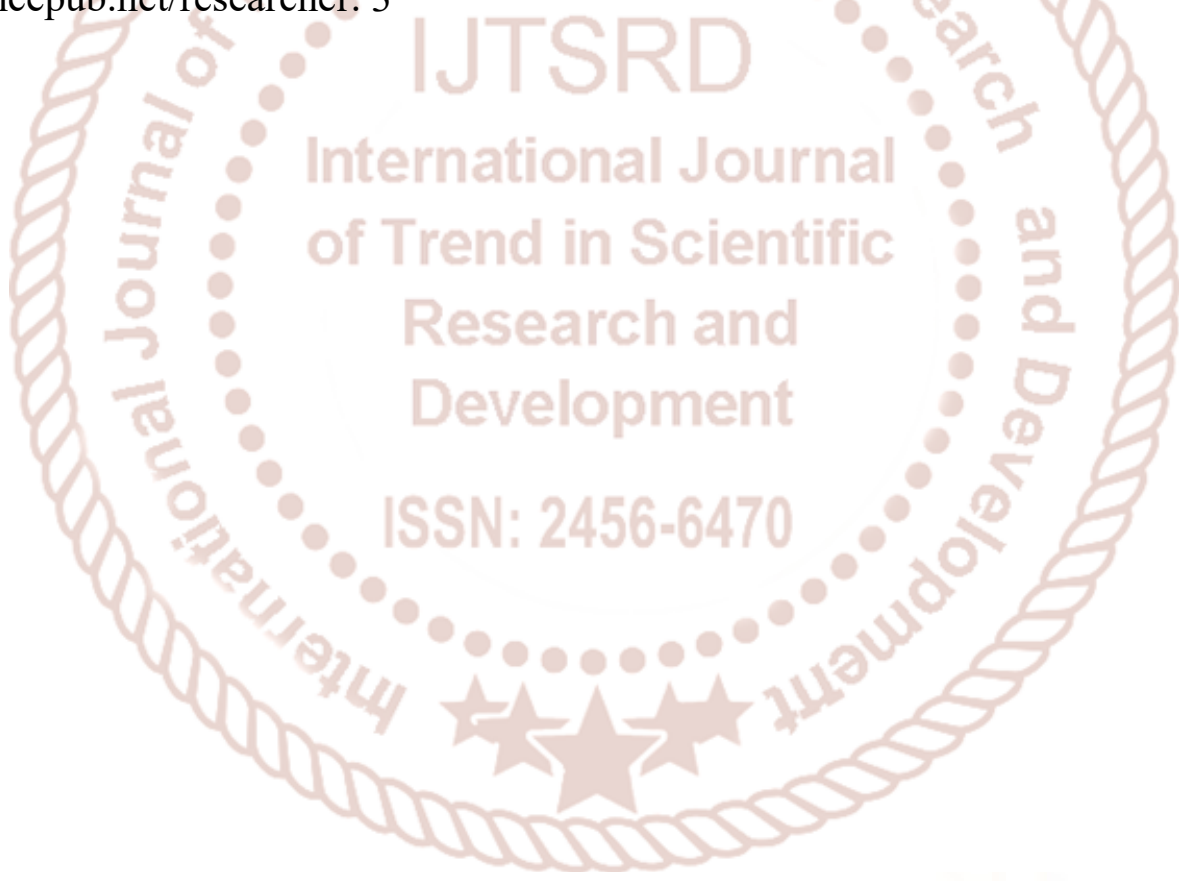

